Supporting Information for:

\title{
Multiplexed Charge Detection Mass Spectrometry for High Throughput Single Ion Analysis of Large Molecules
}

Conner C. Harper, ${ }^{\S}$ Andrew G. Elliott, ${ }^{\S}$ Luke M. Oltrogge, ${ }^{\sharp}$ David F. Savage ${ }^{\sharp}$ and Evan R. Williams ${ }^{\S *}$

\author{
\#Department of Molecular and Cell Biology \\ $\S$ Department of Chemistry \\ University of California, Berkeley, CA 94720
}

For submission to Analytical Chemistry

*Address correspondence to this author.

Email: erw@berkeley.edu

Telephone: (510) 643-7161 


\section{Quadrupole-Time of Flight (Q-TOF) Mass Spectrometry of RuBisCo}

The RuBisCO sample was analyzed using a Waters Micromass Q-TOF mass spectrometer. Mass spectra of the whole complex (Figure S1a) and the large subunit (CbbL, $51.9 \mathrm{kDa}$ ) and small subunit (CbbS, $12.7 \mathrm{kDa}$ ) substituent proteins (S1b) formed from the fragmentation of the whole complex using collision induced dissociation (CID) are shown in Figure S1. A deconvolution of the mass spectrum in S1a is shown in Figure S2. The standard deviation of Gaussian least squares fit to the deconvolved data is $1,700 \mathrm{Da}$, likely due to adduction and incomplete desolvation. This width is smaller than the width of the mass distribution (13 kDa) measured by charge detection mass spectrometry (CDMS), but suggests that at least a portion of the width of the mass peaks in Figure 2 is due to sample heterogeneity and adducts. The additional width of the CDMS mass histogram is likely due to uncertainties in ion energy and charge stemming from varying ion trajectories and the relatively low $\mathrm{S} / \mathrm{N}$ in the Fourier transform for the charge states of RuBisCO $(+48-+52)$. Longer trapping times could therefore decrease the CDMS peak width somewhat; however, sample heterogeneity will ultimately limit the improvement that can be made.

\section{Bovine Serum Albumen (BSA) CDMS measurements}

A mass histogram for BSA containing all data for all trapping events independent of the number of ions that were trapped is shown in Figure S3a. Figure S3b-S3g shows the data for trapping events containing different numbers of ions. Similar to the RuBisCO sample shown in Figure 2, the centroids of each distribution were determined using a Gaussian fit. A linear fit of these centroid data yields and $\mathrm{R}^{2}$ value of 0.0031 , indicating that the measured mass for BSA also does not depend on the number of ions simultaneously 
trapped and analyzed. The centroid values, standard deviations, and average trapping lifetime data for BSA are contained in Table S1. The larger relative standard deviations ( $\sim 5.0 \%$ for all ions) for BSA compared to Rubisco $(\sim 2.5 \%)$ are due to the lower charge of the BSA ions. The uncertainties in the individual ion charge and ion energy measurements both depend on the S/N ratio for signal peaks in the Fourier transform. Lower charge ions have lower S/N and therefore have higher relative uncertainties. Shorter average trapping lifetimes are also observed for BSA compared to RuBisCO. This effect can be attributed to the smaller mass of BSA; smaller ions are more easily destabilized by collisions with background gas and are therefore lost more quickly on average. 
Table S1. Mass and Average Trapping Lifetimes of BSA Obtained by CDMS

Measurements for All Ions and for Events with 1 Through 6+ Simultaneously

Trapped Ions

\begin{tabular}{cccc}
\hline $\begin{array}{c}\text { \# Ions } \\
\text { Simultaneously } \\
\text { Trapped }\end{array}$ & Mass (Da) & $\begin{array}{c}\text { Standard } \\
\text { Deviation of } \\
\text { Gaussian Fit (Da) }\end{array}$ & $\begin{array}{c}\text { Average Trapping } \\
\text { Lifetime (ms) }\end{array}$ \\
\hline All & 66,991 & 3,338 & 573.2 \\
1 & 66,957 & 3,374 & 562.4 \\
2 & 66,968 & 3,358 & 568.8 \\
3 & 67,122 & 3,256 & 574.1 \\
4 & 66,784 & 3,297 & 563.1 \\
5 & 67,318 & 3,225 & 553.6 \\
$6+$ & 66,855 & 3,582 & 569.8 \\
\hline
\end{tabular}




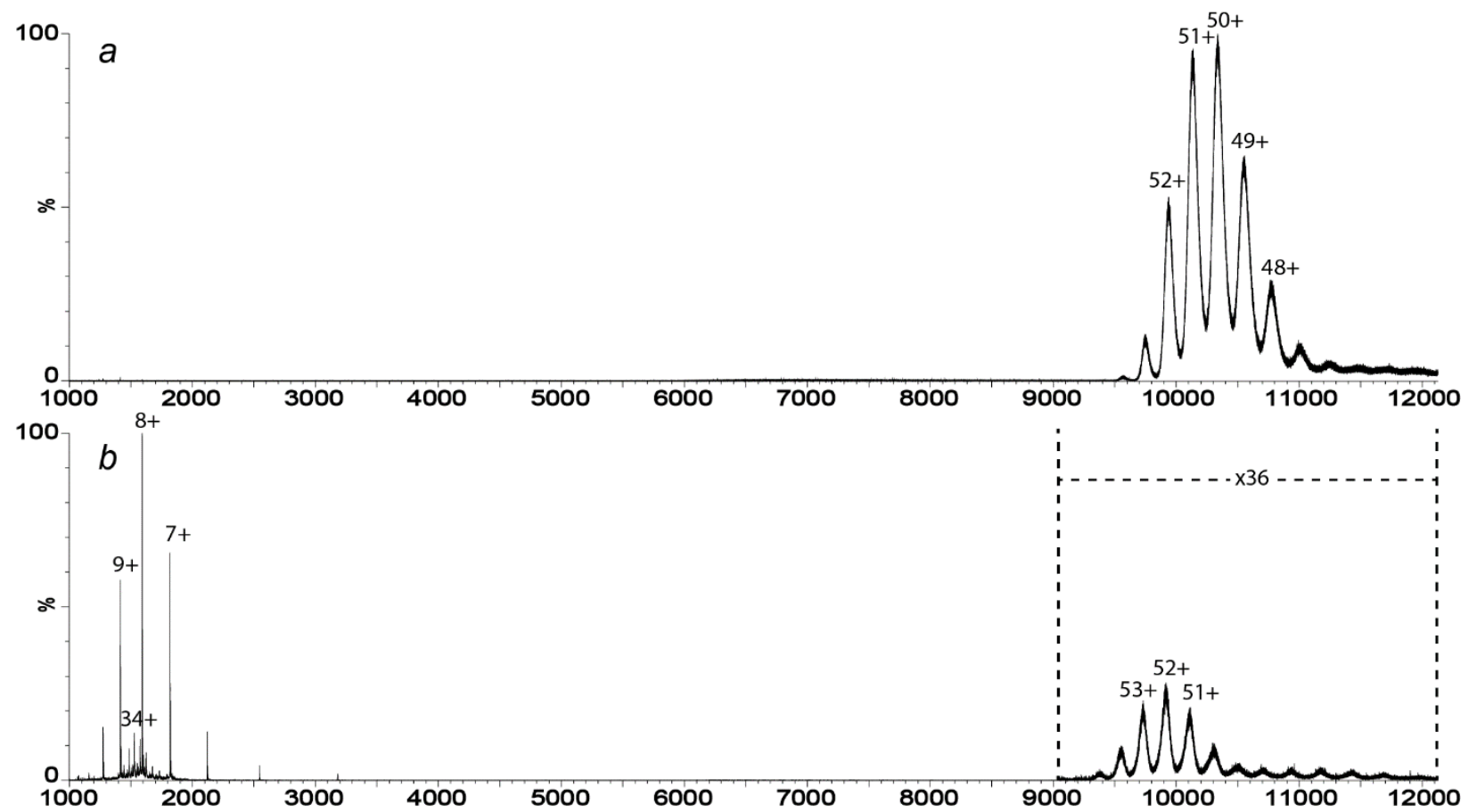

Figure S1. Mass spectra of whole RuBisCO complex before (a) and after (b) CID. The mass determined for RuBisCO in (a, $517 \mathrm{kDa})$ was confirmed by analysis of the small chain $(12.7$ $\mathrm{kDa}, 7+-9+$ labeled) and large chain (51.9 kDa, +34 labeled) CID product ions appearing at lower $m / z$ in (b). 


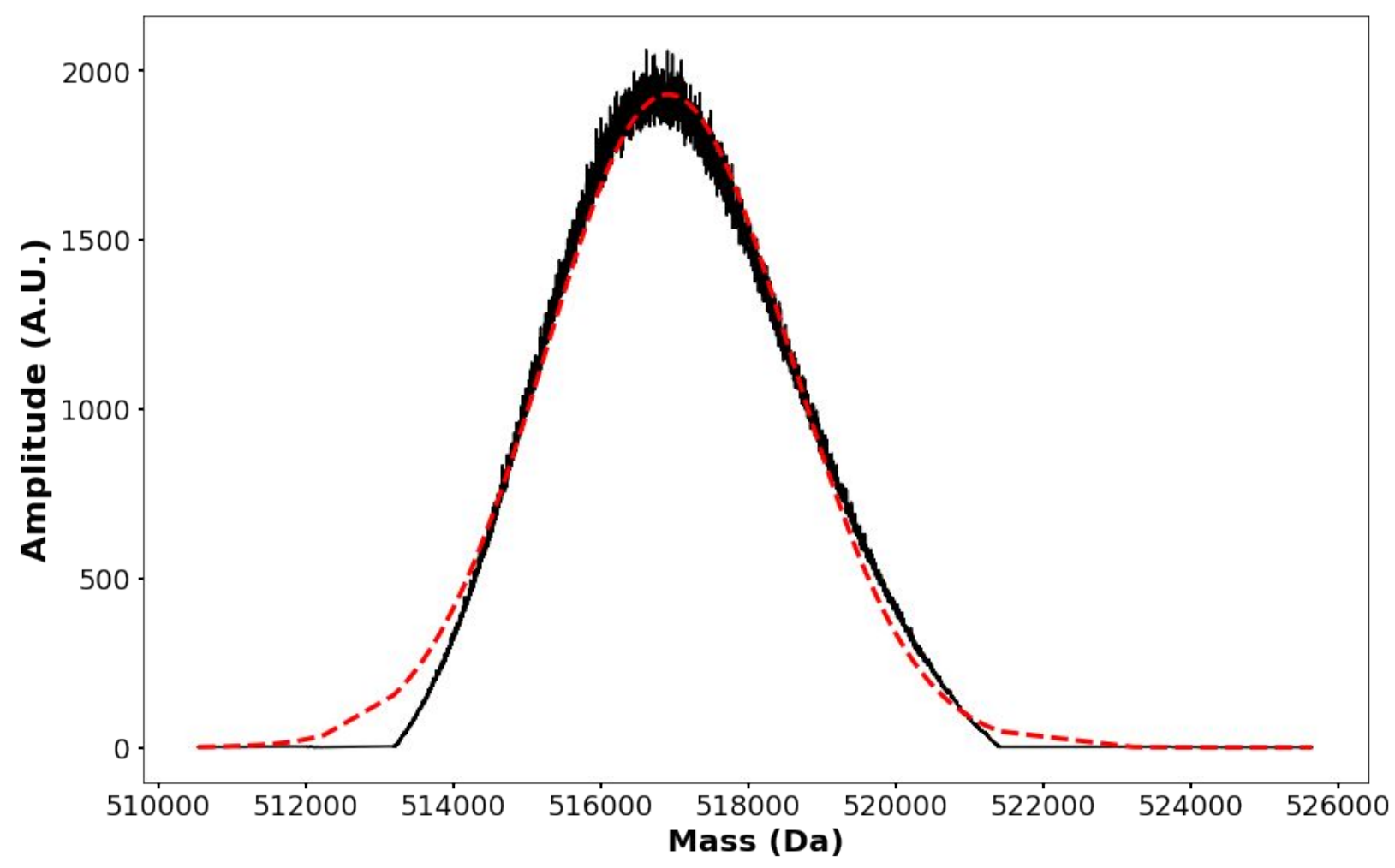

Figure S2. The deconvolved mass spectrum of the data shown in Figure S1a. A Gaussian fit of these data yields a centroid mass of 516,900 $\pm 100 \mathrm{Da}$. The standard deviation of these data is $1,700 \mathrm{Da}$, a width that originates from the intrinsic sample heterogeneity and salt adduction. 

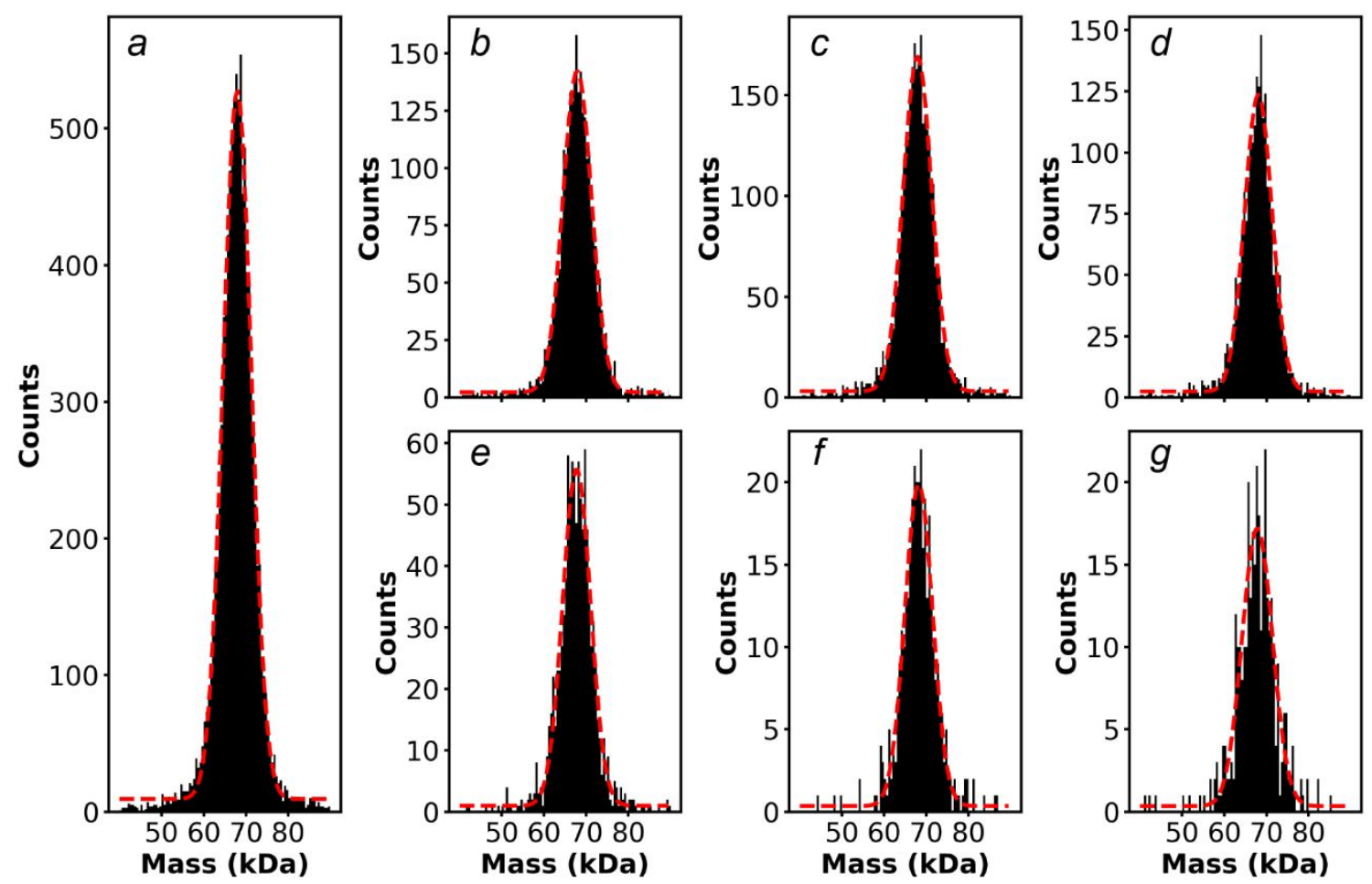

Figure S3. CDMS mass histograms of BSA ions formed by electrospray ionization consisting of (a) all 9637 ions weighed and these data shown separately for ion trapping events where 1 through $6+$ ions were simultaneously trapped (b through $\mathbf{g}$, respectively). A Gaussian fit (red) was used to find the peak centroid corresponding to the reported mass of the complex. A linear fit of the peak centroids for (b) through ( $\mathbf{g}$ ) as a function of number of ions trapped results in $\mathrm{R}^{2}$ value of 0.0031 indicating that the measured mass does not depend on the number of ions that are simultaneously trapped. 